\title{
Calculation of Atomic Structure
}

Jan C. A. Boeyens

\begin{abstract}
The Thomas-Fermi and Hartree-Fock calculations of non-hydrogen atomic structure rely on complicated numerical computations without a simple visualizable physical model. A new approach, based on a spherical wave structure of the extranuclear electron density on atoms, self-similar to prominent astronomical structures, simplifies the problem by orders of magnitude. It yields a normalized density distribution which is indistinguishable from the TF function and produces radial distributions, equivalent to $\mathrm{HF}$ results. Extended to calculate atomic ionization radii it yields more reliable values than SCF simulation of atomic compression. All empirical parameters used in the calculation are shown to be consistent with the spherical standing-wave model of atomic electron density.
\end{abstract}

Keywords: atomic wave model, electron density, golden-spiral optimization, ionization radius, self-similarity

\section{Introduction}

The true meaning of both quantum and relativity theories, which has been demonstrated [1] to emerge only in four-dimensional formalism, has serious implications for the three-dimensional theories of atomic and molecular structure. Nonclassical attributes of atomic matter, such as electron spin, are associated with four-dimensional hypercomplex functions, known as quaternions, and cannot be accounted for by classical three-dimensional models, which include wave mechanics as traditionally formulated. The notorious failure of quantum chemistry to model the structure of non-hydrogen atoms and molecules is a manifestation of the same problem. The awareness that atomic and molecular structure are classical three-

Jan C. A. Boeyens

Unit for Advanced Scholarship, University of Pretoria, South Africa,

e-mail: jan.boeyens@up.ac.za 
dimensional concepts, dictates the use of classical, rather than four-dimensional quantum models, for their characterization.

It is readily demonstrated [2] that a convergence angle of $4 \pi /(2 n-1)$, with integer $n$, generates a set of points on a golden spiral at increasing distances from the origin, reminiscent of the radii, $n^{2} a_{0}$ that occur in the Bohr model of the hydrogen atom. In a related study of satellites in the solar system [3] orbits were found to correspond with divergence angles that optimize the two-dimensional accretion of matter from a rotating uniform cloud, along logarithmic spirals. This consilience can hardly be accidental and accounts for the successful atomic models, independently proposed by Nagaoka and Bohr, based on the structure of Saturnian rings and planets in the solar system, respectively.

Although spectacularly successful at the time, these early atomic models failed, because they followed the two-dimensional astronomical analogues, which are characterized by three-dimensional angular-momentum vectors, directed along fixed rotation axes, too closely. Electronic rotation, by contrast, occurs in spherical mode, correctly described by quaternions, that give rise to the quantum-mechanical spin function. The resulting distribution of extranuclear negative charge occurs in shells around the atomic nucleus, in the form of a standing spherical wave. Although the total charge is an integral multiple of the elementary electronic charge, individual electrons cannot be distinguished within the undulating electric fluid. There are, in particular, no such things as electronic particles in this model. The elementary charge is associated with an elementary wave packet, which on further sub-division, through interaction with a positron, disperses into the vacuum.

The Bohr atomic model, which describes an electron as an orbiting particle, is well known to fail for all atoms other than hydrogen. Maxima in the optimization function should therefore not be interpreted as orbits, but rather as the nodes of a spherical standing wave in line with the periodic table of the elements.

\section{The Periodicity of Matter}

It was first noted by William Harkins [4] that the ratio $(A-Z) / A$ for the known nuclides never exceeded 0.62 , later identified more accurately as the golden ratio, $\tau=0.61803 \cdots=\Phi^{-1}$ [5]. A plot of $Z / N v s A$ of non-radioactive nuclides are shown in Fig. 1 with convergence curves as inferred by Harkins.

The region of stability is mapped more precisely by two sets of straight-line segments with inflection points at common values of $A$. Through these inflection points eleven hem lines divide the field of stability such that each block contains 24 nuclides. Although there is no general agreement on half lives that define stable nuclides, the set of nuclides identified by different schemes never deviates seriously from the 264 selected in Fig. 1.

Replotting the data on axes of $Z / N$ vs $Z$ (Fig. 2) does not affect the general shape of the straight-line profiles, but the hem lines, because of their modified slopes, no longer intercept all lines of constant $Z / N$ at the same $Z$. 


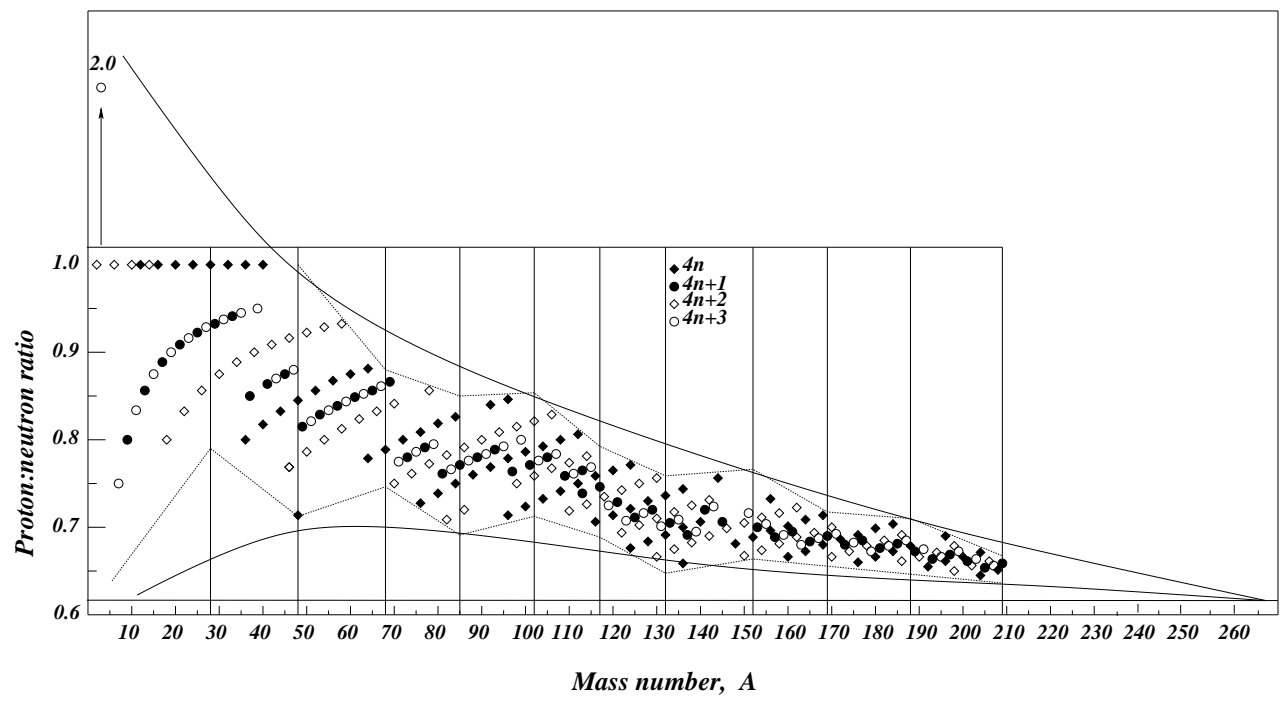

Fig. 1 Distribution of stable nuclides as a function of mass number

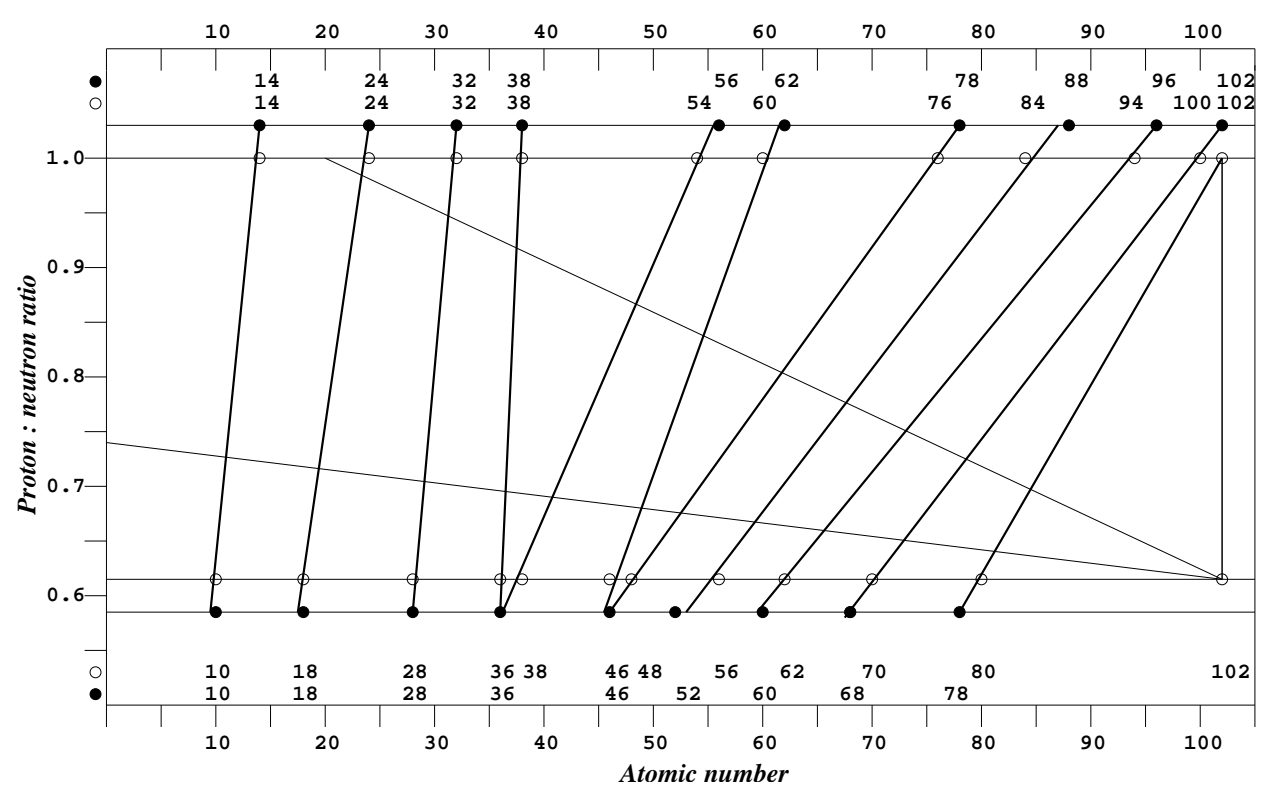

Fig. 2 Periodic functions defined by nuclide distribution

Intersection with the lines $Z / N=0.58,0.62,1.0$ and 1.04 is of special interest. The points of intersection along $Z / N=\tau$ all coincide with atomic numbers commonly interpreted to signal the completion of an electronic subshell according to the 
periodic table of the elements:

$$
10(2 p), 18(3 p), 28(3 d), 36(4 p), 38(5 s), 46(4 d), 48(5 s), 56(6 s), 62(4 f: 6 / 8), 70(4 f), 80(5 d)
$$

The points at 0.58 define the periodicity implied by the wave-mechanical solution of the $\mathrm{H}$ electron:

$$
10(2 p), 18(3 p), 28(3 d), 36(4 p), 46(4 d), 52(4 f: 6 / 8), 60(4 f), 68(5 p), 78(5 d)
$$

The relationship with the points of intersection at $N / Z=1.0$ and at $N / Z=1.04$ is clarified by noting how these points represent an inversion of energy levels. The points at 1.04:

$$
14(4 f), 24(3 d), 32(1 s), 38(5 f: 6 / 8), 56(4 d), 62(3 p), 78(6 f), 88(5 d), 96(3 s)
$$

represent the completely inverted wave-mechanical spectrum $4 f<3 d<2 p<$ $1 s \ldots$... etc. The points at 1.0 define the inverted observed periodic table of the elements.

The only known process which could invert atomic energy levels is the application of relentless pressure [6]. It becomes logical to imagine that inverted periodicity occurs where the environmental pressure on an atom approaches infinity, as in a black hole. The reciprocal situation of zero pressure correlates with the wavemechanical assumption of nothing but the potential field of a single proton. This implies an empty universe and therefore flat space-time. By this argument the arrangement at $N / Z=\tau$ occurs at the moderate curvature and pressure that prevails in the solar system. At $Z / N=1$ an equilibrium between protons and neutrons is interpreted as suitable conditions for high-pressure synthesis of all nuclides from ${ }^{4} \mathrm{He}$.

This interpretation implies a periodicity of 24 among stable nuclides, with the periodic table of the elements as a subset. It confirms that the observed periodicity is not predicted correctly by wave mechanics, but can be derived without the use of higher mathematics. The prominent role of $Z / N=\tau$ suggests a possible numbertheory model of elemental periodicity.

\subsection{The Periodic Table}

The important $Z / N$ ratio must by definition always be a rational fraction and an ordered set of nuclides must therefore correspond to a Farey sequence. It is readily demonstrated [7] that a set of $k$-modular Simple Farey fractions

$$
S_{k}=\frac{n}{n+k},
$$

not necessarily in reduced form, plotted against natural numbers, has the same appearance as Fig. 1, except for being of infinite extent. Convergence of nuclide com- 
position from unity to $Z / N \rightarrow \tau$ implies that the limiting curves must be generated by the intersection of the infinite Farey festoons with a converging series of Fibonacci fractions. This way it could be demonstrated [6] that the points within the resulting triangle of stability represent the naturally occurring stable isotopes.

The relationship between unimodular Farey sequences and Ford circles [8] enables direct mapping of the periodic function by touching Ford circles, producing a table of the form shown in Fig. 3 [8].

\begin{tabular}{|c|c|c|c|c|c|c|}
\hline 8788 & \begin{tabular}{l|l}
94 & 95 \\
\end{tabular} & \begin{tabular}{l|l}
102 & 103 \\
\end{tabular} & 110 & 111 & 118 & 32 \\
\hline & & \begin{tabular}{|l|l|}
3738 & 39 \\
\end{tabular} & 46 & 4748 & 54 & 18 \\
\hline & & & & 1111 & 18 & 8 \\
\hline & & & & & $\begin{array}{ll}1 & 2 \\
\end{array}$ & 2 \\
\hline & & & & 34 & 10 & 8 \\
\hline & & \begin{tabular}{|l|l|}
1920 & 21 \\
\end{tabular} & 28 & 2930 & 36 & 18 \\
\hline 5556 & \begin{tabular}{l|l}
62 & 63 \\
\end{tabular} & $\begin{array}{lll}70 & 71\end{array}$ & 78 & 7980 & 86 & 32 \\
\hline
\end{tabular}

Fig. 3 Mapping of the periodic table of the elements as the reciprocal radii of the $\mathscr{F}_{4}$ unimodular Ford circles

The characteristic values of $Z / N=\tau$, and of 0.58 for observed and wavemechanical periodicities are the limits of converging Fibonacci fractions around $3 / 5$. The segmentation of the table into groups of 2 and 8 and of periods $2,8,18,32$ summarizes the observed periodicity as a subset of nuclide periodicity. The sub-level structure, despite formal resemblance to the wave-mechanical $\mathrm{H}$ solution, emerges from number theory without reference to atomic structure.

We now consider the possibility of characterizing the electronic structure of atoms as it relates to cosmic self-similarity and the periodicity of atomic matter.

\section{The Golden Spiral}

Casual interpretation of the local environment as three-dimensional space and universal time flow is not consistent with the known four-dimensional structure of space-time on a cosmic scale. Local Euclidean space is said to be tangent to the underlying four-dimensional curved space-time.

It has been argued [8] that the transformation from curved space-time to Euclidean tangent space is described by the golden ratio. This is not an entirely unexpected conclusion, in view of the prominence of $\tau$ in the operation of self-similar symmetries, related to equiangular logarithmic spirals.

The complex number $(a, b)=a+i b$ is represented in polar coordinates by $(r, \theta)=r e^{i} \theta=r(\cos \theta+i \sin \theta)$ as in Fig. 4. Continuous rotation of the point $(a, 0)$, which transforms each point $(r, \theta)$ into $(r, \theta+t)$, has the locus $r=a$ that describes a circle. In the same way, when $(a, 0)$ is transformed by continuous dilatation (enlargement), the locus $\theta=0$ of $(a, 0)$ describes the ray $(r, 0)$ for increasing $r>0$. 
Fig. 4 The complex plane

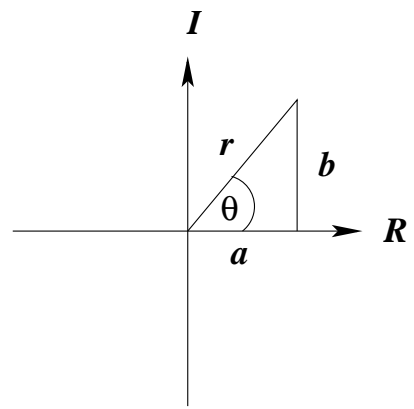

Combination of these two operations represents a continuous dilative rotation that transforms the general point $(r, \theta)$ into $\left(\alpha^{t} r, \theta+t\right)$, as $t$ changes continuously. The locus of the transform of $(a, 0)$ is the equiangular spiral

$$
r=\alpha^{t} a \quad, \quad \theta=t
$$

i.e. $r=a \mu^{\theta}$. Taking the derivative:

$$
\begin{aligned}
\frac{d r}{d \theta} & =\frac{d}{d \theta}\left(a \mu^{\theta}\right) \\
& =a \mu^{\theta} \ln \mu=r \ln \mu
\end{aligned}
$$

The constant $\mu$ represents the dilatation on rotation of 1 radian. On rearrangement into

$$
\frac{d r}{r}=d \ln r=\ln \mu d \theta,
$$

the simplest equation of the logarithmic spiral follows as $r=\mu \exp (c \theta)$, more frequently given in the classical form

$$
r=a e^{\theta \cot \phi}
$$

where $a$ and $\phi$ are constants. It is common practice to set $a=1$ to obtain a unit spiral.

In the special case where $\phi \simeq 73^{\circ}, \cot \phi=\tau / 2$, the spiral $r=a \exp (\theta \tau / 2)$, equivalent to $r=a / \tau^{2 \theta / \pi}$, corresponds almost exactly to the golden spiral [9], as constructed in a golden rectangle, and shown in Fig. 5. The constant $a$ is related to the overall dimensions of the spiral [10]. More generally, in terms of the complex number $a+i b$ :

$$
r=A e^{(a / b) \theta} \quad, \quad b \neq 0 .
$$

The distinctive property of logarithmic spirals is the constant dilatation for equal rotations. A dilative rotation of $2 \pi$ transforms any point on the spiral into a homothetic point, which is similarly placed and directed. The spiral, said to be homothetic to itself, therefore has the property of self-similarity at all scales. We note that the 
Fig. 5 The approximate golden logarithmic spiral. $\tan \alpha=\tau$. Note that $\phi \simeq 2 \pi / 5$ radians, emphasizing the relationship of $\tau$ to a regular pentagon: $\cos (2 \pi / 5)=\tau / 2$

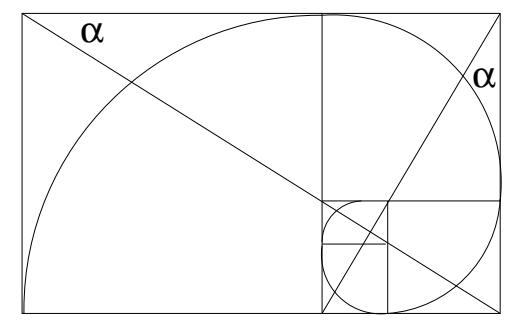

origin $(r, \theta=0)$ transforms into the homothetic points $e^{n \pi \tau}$ after $n$ rotations. We propose that this property, described by the three fundamental constants: $e, \pi$ and $\tau$, is related to the general curvature of space-time, which is responsible for the observed cosmic self-similarity: $e$ for growth, $\pi$ for rotation and $\tau$ for dilatation.

\subsection{Self-similarity}

The demonstration [1] that both Lorentz transformation and quantum spin are the direct result of quaternion rotation implies that all relativistic and quantum structures must have the same symmetry. This is the basis of cosmic self-similarity. The observation that the golden mean features in many known self-similarities confirms that $\tau$ represents a fundamental characteristic of space-time curvature. The existence of antimatter and the implied $\mathrm{CPT}^{1}$ symmetry of space-time favours closed metric-free projective geometry with involution; the only topology that automatically generates the gauge invariance that links quantum mechanics to the electromagnetic field [11]. This topology is consistent with constant space-time curvature, locally distorted by large gravitating masses. It seems reasonable to assume that the logarithmic spiral (1) follows the general curvature in two-dimensional projection, characteristic of stable structures and growth patterns in tangent Euclidean space. In four-dimensional space-time the curvature is more appropriately described by a formula such as

$$
\rho\left(x^{\mu}\right)=A e^{\left(a / \sqrt{b^{2}+c^{2}+d^{2}}\right) \theta},
$$

which describes spherical rotation in quaternion notation.

The spacing of planetary orbits and of moons orbiting a planet have been shown [3] to obey simple whole number rules, not unlike the quantum rules of wave mechanics. Orbital radii, in particular, correspond to positions separated by a constant divergence angle along a golden spiral. We now demonstrate that the spacing of atomic electron shells are also related by a divergence angle, according to the same procedure.

\footnotetext{
${ }^{1}$ Charge conjugation-parity-time
} 


\section{Atomic Structure}

Each electronic energy level is assumed to occur as a spherical shell at a distance from the nucleus, determined by a positive integer $n$ and a minimum radius $a$ of the innermost shell. Atomic size is wave-mechanically poorly defined. For principal quantum number $n$, the electronic radial distribution function has a single maximum [13] when the angular momentum quantum number $l$, has its largest value of $n-1$. The relative density is determined by the degeneracy of $2 l+1=2 n-1$. Enhanced nuclear attraction and increased charge density, imply that divergence angles for optimal spacing will not be constant, but likely to decrease by the factor $(2 n-1)$ for each quantum level. The first shell is assumed to occur at a rotation of $4 \pi$ from the origin $^{2}$. Successive divergence angles for higher levels then follow as $\phi_{n}=4 \pi /(2 n-$ $1)$.

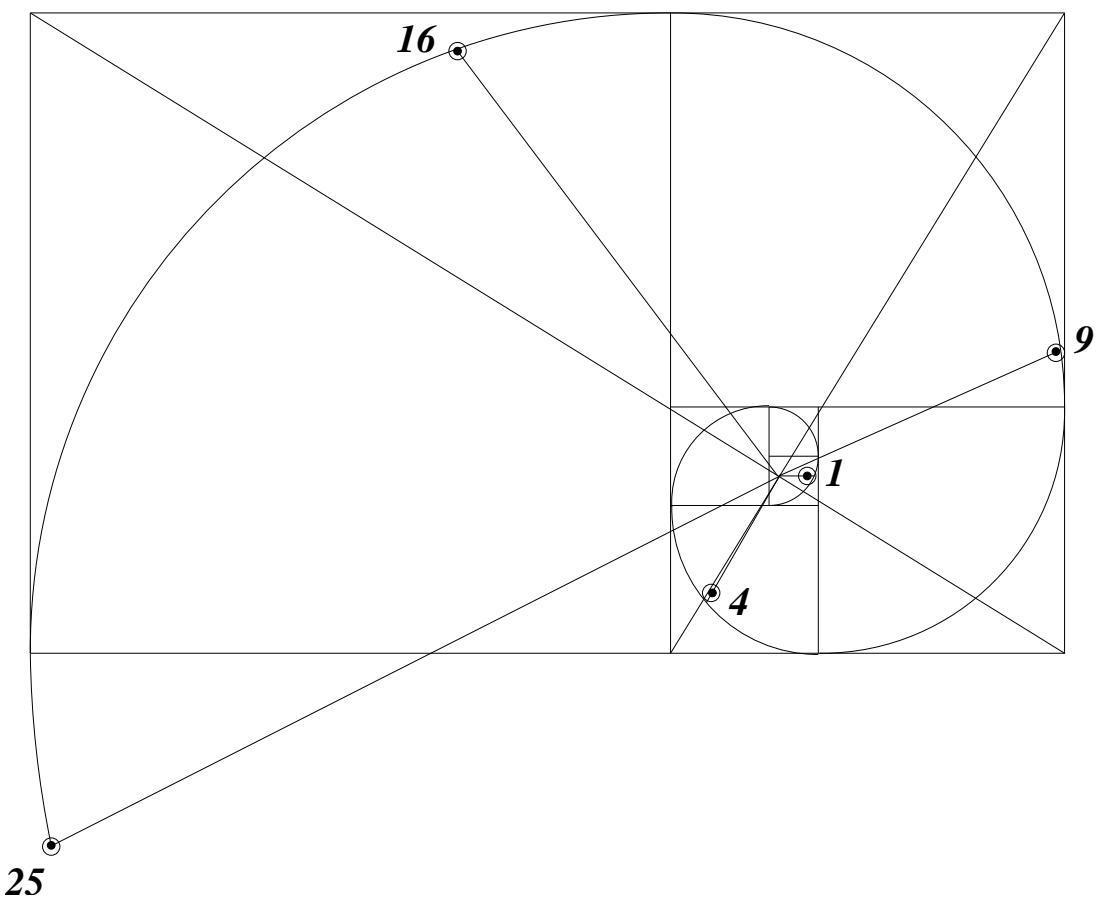

Fig. 6 A sequence of Fibonacci squares on a scale of 1:2 serves to generate the $21 \times 13 \mathrm{~cm}$ golden rectangle with its inscribed spiral. Directly measurable radii of $n^{2} a(a=6.7 \mathrm{~mm})$ at convergence angles of $4 \pi /(2 n-1)$ terminate at the labeled points.

Using this as a guide the distribution of charge density, optimized by a golden spiral at a divergence angle of $4 \pi /(2 n-1)$, for principal quantum number $n$, was

\footnotetext{
2 This generates spin of $\hbar / 2$.
} 
Table 1 The Schrödinger Table

$\begin{array}{cccc}n & \text { Configuration } & \text { Electrons } & \text { Total } \\ 1 & 1 s^{2} & 2 & 2 \\ 2 & 2 s^{2} 2 p^{6} & 8 & 10 \\ 3 & 3 s^{2} 3 p^{6} 3 d^{10} & 18 & 28 \\ 4 & 4 s^{2} 4 p^{6} 4 d^{10} 4 f^{14} & 32 & 50 \\ 5 & 5 s^{2} 5 p^{6} 5 d^{10} 5 f^{14} 5 g^{18} & 50 & 100\end{array}$

calculated before [2]. The simplest demonstration of such electron-density optimization is in terms of a Fibonacci spiral [8] that converges to a golden spiral with increasing Fibonacci number. Graphical derivation of orbital radii, according to this model, is shown in Fig. 6, predicting $r / a=1,4,9,16$, etc., for unit radius $a$, in agreement with the Bohr radii of $r_{n}=n^{2} a_{0}$. The labeled points in Fig. 6 lie progressively closer to the spiral and predict to good approximation the volumes and charge densities of al atoms with $Z=1 \rightarrow 118$. On a virtually identical logarithmic spiral, $r=1.164 \exp (\theta \cdot \tau / 2)$, when sampled at $\theta_{n}=\sum_{n} 4 \pi /(2 n-1)$, the $r_{n}$ round off to $n^{2}$.

According to Schrödinger's solution for the hydrogen atom and the exclusion principle, successive energy levels can accommodate $2 n^{2}$ electrons, as in Table 1 . This arrangement cannot account for either the periodic table of the elements, the electronic structure of non-hydrogen atoms or the predicted orbital radii.

Fig. 7 Self-similar distribution of atomic electron density. For $Z=1$ the unit of radial distance is assumed as $a=a_{0}$, the Bohr radius

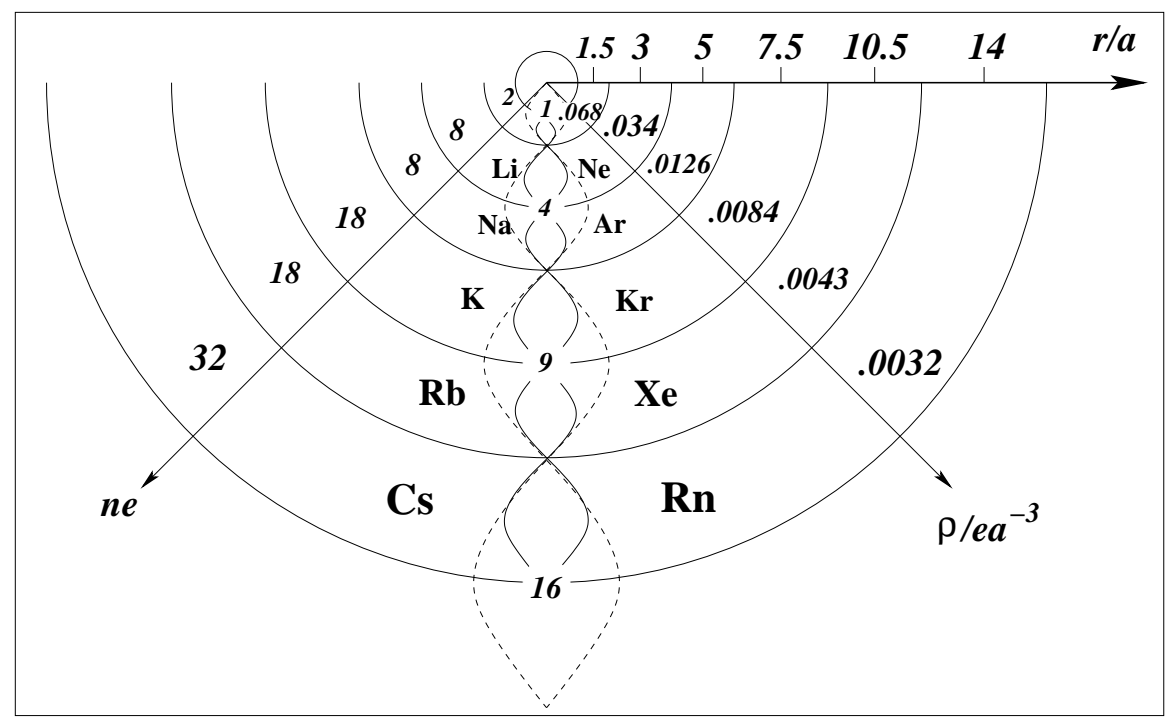


The Thomas-Fermi (TF)[14] and Hartree-Fock (HF) schemes model atomic structure empirically, by assuming spectroscopically determined electron configurations. On the other hand, the correct form of the periodic table emerges from elementary number theory, which suggests an alternative interpretation of the Bohr radii.

Table 2 Screening factors $(v)$ and comparison of the calculated maxima at alkali-metal wave crests $\left(\bar{r}_{n}\right)$, scaled against Hartree-Fock [16] radial expectation values, in units of $a_{0}$.

\begin{tabular}{|c|c|c|c|c|c|c|c|c|}
\hline$n$ & $r_{n}^{\prime} / a_{0}$ & $\mathrm{Li}$ & $\mathrm{Na}$ & K & $\mathrm{Rb}$ & Cs & $\mathrm{Fr}$ & Key \\
\hline 1 & 1.5 & $\begin{array}{c}1 \\
0.50 \\
0.57\end{array}$ & $\begin{array}{c}1 \\
0.136 \\
0.143\end{array}$ & $\begin{array}{c}1 \\
0.079 \\
0.081\end{array}$ & $\begin{array}{c}1 \\
0.041 \\
0.041\end{array}$ & $\begin{array}{c}1 \\
0.027 \\
0.028\end{array}$ & $\begin{array}{c}1 \\
0.017 \\
0.017\end{array}$ & $\begin{array}{l}v_{i} \\
\bar{r}_{n} \\
\mathrm{HF}\end{array}$ \\
\hline 2 & 3 & $\begin{array}{l}4.05 \\
3.87\end{array}$ & $\begin{array}{c}3 \\
0.818 \\
0.779\end{array}$ & $\begin{array}{c}2 \\
0.316 \\
0.386\end{array}$ & $\begin{array}{c}2 \\
0.162 \\
0.169\end{array}$ & $\begin{array}{c}2 \\
0.109 \\
0.110\end{array}$ & $\begin{array}{c}2 \\
0.069 \\
0.068\end{array}$ & \\
\hline 3 & 5 & & $\begin{array}{l}4.50 \\
4.21\end{array}$ & $\begin{array}{c}5 \\
1.315 \\
1.357\end{array}$ & $\begin{array}{c}4 \\
0.540 \\
0.520\end{array}$ & $\begin{array}{c}3 \\
0.273 \\
0.297\end{array}$ & $\begin{array}{c}3 \\
0.172 \\
0.168\end{array}$ & \\
\hline 4 & 7.5 & & & $\begin{array}{l}5.07 \\
5.24\end{array}$ & $\begin{array}{c}7.5 \\
1.620 \\
1.617\end{array}$ & $\begin{array}{c}5 \\
0.682 \\
0.768\end{array}$ & $\begin{array}{c}5 \\
0.431 \\
0.392\end{array}$ & \\
\hline 5 & 10.5 & & & & $\begin{array}{l}5.67 \\
5.63\end{array}$ & $\begin{array}{c}10.5 \\
2.005 \\
1.975\end{array}$ & $\begin{array}{c}7.5 \\
0.905 \\
0.916\end{array}$ & \\
\hline 6 & 14 & & & & & $\begin{array}{l}6.31 \\
6.31\end{array}$ & $\begin{array}{c}14 \\
2.253 \\
2.168\end{array}$ & \\
\hline 7 & 18 & & & & & & $\begin{array}{l}6.95 \\
6.63\end{array}$ & \\
\hline
\end{tabular}

Interpreted in terms of the symmetrical form of the periodic table (Fig. 3) the quantum numbers that define the radial distances of $r=n^{2} a$ specify the nodal surfaces of spherical waves that define the electronic shell structure. Knowing the number of electrons in each shell, the density at the crests of the spherical waves that represent periodic shells, i.e. at $1.5,3$, etc. $(a)$, can be calculated. This density distribution, shown in Fig. 7, decreases exponentially with $Z$ and, like the TF centralfield potential, is valid for all atoms and also requires characteristic scale factors to generate the density functions for specific atoms. The Bohr-Schrödinger model, represented by the stippled curve in Fig. 7, breaks down completely for non-hydrogen atoms.

The TF potential is shown as a solid curve [14] in Fig. 8, with our calculated points on the same scale (Numerical factor=55). The curve in stippled outline is an approximate simulation of the Hartree-Fock electron density for unit atoms. A few simple assumptions allow more detailed simulation of HF results for any atom: 


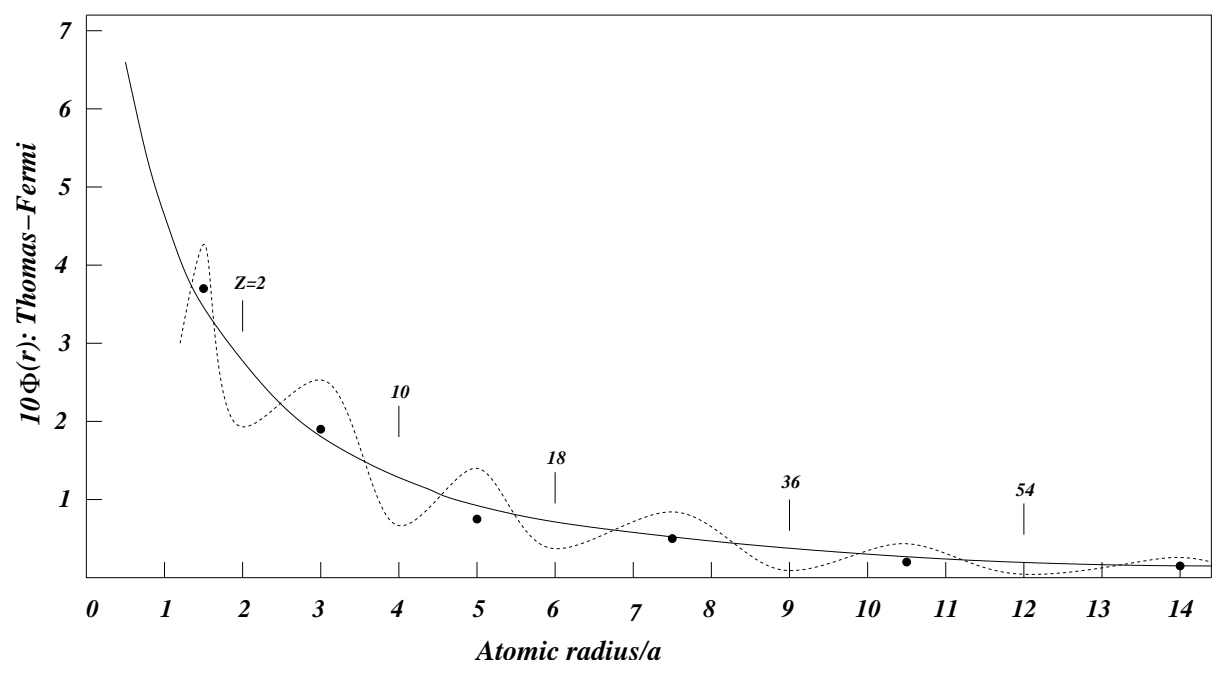

Fig. 8 Simulation of Thomas-Fermi and Hartree-Fock electron densities for unit atoms

(i) An electron at the innermost level is not screened against attraction by the nuclear charge of $+Z e$. The one-electron radius $r^{\prime}=1.5 a_{0}$ is thereby contracted to $r_{1}=r^{\prime} / Z[15]$.

(ii) The radii of intermediate shells contract to

$$
r_{i}=\frac{v_{i} r_{n}^{\prime}}{Z}
$$

where $v$ is a screening constant.

(iii) The radius of the outer shell is modelled as

$$
r_{n}^{\prime \prime}=\frac{r_{n}^{\prime}}{n s} .
$$

Using the HF results of Mann [16] for the alkali metals as a benchmark, this screening factor could be fixed at $s=0.37$. Appropriate values of $v_{i}$ are summarized in Table 2.

In this calculation the calculated maxima $\bar{r}_{n}$ are scaled against the radial expectation values of $\mathrm{Mann}^{3}$. The multiplet level structure of the HF analysis is reproduced in detail. The fundamental assumption underlying this simple simulation is the indistinguishability of individual electrons in a collective, as emphasized by Schrödinger [17], Madelung [18] and Pauli [19]. This calculation has not been done for other

${ }^{3} \mathrm{SCF}$ multiplet structure is empirically based on spectroscopic results 
elements, but once outer-level radial expectation values had been obtained, the procedure of Table 2 applies.

The highest radial maxima for non-alkali atoms in the same period must decrease uniformly from the alkali values $r_{n}^{\prime \prime}$, depending on the number of electrons at each sub-level. For elements of the $p$-block correct values of these radial maxima are predicted as

$$
\bar{r}=\frac{r_{n}^{\prime \prime} \cdot \sigma}{\mu^{p}}
$$

from characteristic values of $\sigma$ and $\mu$ for a number $p$ of electrons. The results are summarized in Table 3.

Table 3 Calculated radii $\bar{r} / a_{0}$ of highest electron-density maxima of $p$-block and alkaline-earth elements, compared to the corresponding HF values [16]

\begin{tabular}{|c|c|c|c|c|c|c|c|c|c|}
\hline$\mu$ & $\sigma$ & $r_{n}^{\prime \prime}$ & \multicolumn{70}{|c|}{$\bar{r}$} \\
& & & \multicolumn{7}{|c|}{} \\
\hline 1.17 & & $\mathrm{Li}$ & $\mathrm{Be}$ & $\mathrm{B}$ & $\mathrm{C}$ & $\mathrm{N}$ & $\mathrm{O}$ & $\mathrm{F}$ & $\mathrm{Ne}$ \\
& 0.59 & 4.05 & 3.27 & 2.04 & 1.75 & 1.49 & 1.27 & 1.09 & 0.93 \\
& $(\mathrm{HF})$ & 3.87 & 2.65 & 2.20 & 1.74 & 1.45 & 1.24 & 1.08 & 0.97 \\
\hline 1.14 & & $\mathrm{Na}$ & $\mathrm{Mg}$ & $\mathrm{Al}$ & $\mathrm{Si}$ & $\mathrm{P}$ & $\mathrm{S}$ & $\mathrm{Cl}$ & $\mathrm{Ar}$ \\
& 0.8 & 4.50 & 3.62 & 3.16 & 2.77 & 2.43 & 2.13 & 1.87 & 1.64 \\
& $(\mathrm{HF})$ & 4.21 & 3.25 & 3.43 & 2.79 & 2.37 & 2.07 & 1.84 & 1.66 \\
\hline 1.11 & & $\mathrm{~K}$ & $\mathrm{Ca}$ & $\mathrm{Ga}$ & $\mathrm{Ge}$ & $\mathrm{As}$ & $\mathrm{Se}$ & $\mathrm{Br}$ & $\mathrm{Kr}$ \\
& 0.7 & 5.07 & 4.09 & 3.20 & 2.88 & 2.59 & 2.34 & 2.11 & 1.90 \\
& $(\mathrm{HF})$ & 5.24 & 4.21 & 3.42 & 2.90 & 2.56 & 2.31 & 2.11 & 1.95 \\
\hline 1.11 & & $\mathrm{Rb}$ & $\mathrm{Sr}$ & $\mathrm{In}$ & $\mathrm{Sn}$ & $\mathrm{Sb}$ & $\mathrm{Te}$ & $\mathrm{I}$ & $\mathrm{Xe}$ \\
& 0.73 & 5.67 & 4.57 & 3.73 & 3.36 & 3.03 & 2.73 & 2.45 & 2.21 \\
& $(\mathrm{HF})$ & 5.63 & 4.63 & 3.78 & 3.29 & 2.95 & 2.70 & 2.50 & 2.34 \\
\hline 1.10 & & $\mathrm{Cs}$ & $\mathrm{Ba}$ & $\mathrm{Tl}$ & $\mathrm{Pb}$ & $\mathrm{Bi}$ & $\mathrm{Po}$ & $\mathrm{At}$ & $\mathrm{Rn}$ \\
& 0.68 & 6.31 & 5.09 & 3.90 & 3.54 & 3.22 & 2.93 & 2.67 & 2.42 \\
& $(\mathrm{HF})$ & 6.31 & 5.26 & 3.93 & 3.45 & 3.14 & 2.89 & 2.70 & 2.54 \\
\hline & & $\mathrm{Fr}$ & $\mathrm{Ra}$ & & & & & & \\
& & 6.95 & 5.60 & & & & & & \\
\hline
\end{tabular}

The parameters $\sigma$ and $1 / \mu$ are empirical estimates which describe the stepwise radial decrease of $s-$ and $p$-density maxima within a periodic group. The expected periodic increase of $\sigma$ with increasing period number is interrupted by atomic contraction due to the first appearance of $d$ and $f$ levels. It will be shown that these parameters depend on the periodic variation of angular momentum and spin, and hence the exclusion principle.

The decrease of $\mu$ with periodic number refers to the relative weight of a single electron in shells of different size. At an $s$ level, consisting of only two electrons, we estimate $\mu=2 \tau$, decreasing smoothly and converging to $\mu=1$ with increasing electron count. Accordingly we calculate $\bar{r}=r^{\prime \prime} / 2 \tau$ for the alkaline-earth metals, 
also shown in Table 3 . The decrease of $\mu(s)>\mu(p)>\mu(d)>\mu(f) \rightarrow 1$, reflects the same trend.

All atoms, except for those of the $p$-block and of the element palladium, have an outer shell of $s$ electrons. Atoms of the so-called $d$-block have a penultimate $d$-shell. Variation of atomic radius, within such a series with a uniform outer shell, is almost continuous. Discontinuity occurs where the number of electrons in the outer shell differs from the general $s^{2}$.

The $d$-block, consisting of the $3 \times 8$ transition elements and the coinage group, has radial expectation values described correctly as

$$
\bar{r}=\frac{r_{n}^{\prime \prime} \sigma}{(1.03)^{d}}, \quad \text { and } \quad \bar{r}=\frac{r_{n}^{\prime \prime} \sigma}{(1.01)^{f}}
$$

describes inner-transition elements with an incomplete $f$-shell. The results are in Tables 4 and 5.

Table 4 Radial distances of highest maxima of d-block elements

\begin{tabular}{|c|c|c|c|c|c|c|c|c|c|c|c|}
\hline$\sigma$ & $r_{n}^{\prime \prime}$ & $\mathrm{Sc}$ & $\mathrm{Ti}$ & V & $\mathrm{Cr}^{*}$ & Mn & $\mathrm{Fe}$ & $\mathrm{Co}$ & $\mathrm{Ni}$ & $\mathrm{Cu}^{*}$ & $\mathrm{Zn}$ \\
\hline \multirow{3}{*}{$\begin{array}{l}0.78 \\
0.88\end{array}$} & 5.07 & 3.84 & 3.73 & 3.62 & & 3.41 & 3.31 & 3.22 & 3.12 & & 2.94 \\
\hline & & & & & 3.85 & & & & & 3.32 & \\
\hline & $(\mathrm{HF})$ & 3.96 & 3.77 & 3.69 & 3.84 & 3.35 & 3.24 & 3.16 & 3.06 & 3.33 & 2.90 \\
\hline \multirow{4}{*}{$\begin{array}{l}0.76 \\
0.85\end{array}$} & 5.67 & $\mathrm{Y}$ & $\mathrm{Zr}$ & $\mathrm{Nb}^{*}$ & $\mathrm{Mo}^{*}$ & $\mathrm{Tc}$ & $\mathrm{Ru}^{*}$ & $\mathrm{Rh}^{*}$ & $\mathrm{Pd}^{* *}$ & $\mathrm{Ag}^{*}$ & $\mathrm{Cd}$ \\
\hline & & 4.18 & 4.06 & & & 3.72 & & & & & 3.21 \\
\hline & & & & 4.28 & 4.16 & & 3.92 & 3.80 & $(4.2)$ & 3.59 & \\
\hline & (HF) & 4.30 & 4.08 & 4.21 & 4.08 & 3.65 & 3.88 & 3.80 & 1.53 & 3.66 & 3.24 \\
\hline \multirow{4}{*}{$\begin{array}{l}0.69 \\
0.78\end{array}$} & 6.31 & $\mathrm{Lu}$ & $\overline{\mathrm{Hf}}$ & $\mathrm{Ta}$ & $\mathrm{W}$ & $\mathrm{Re}$ & Os & $\mathrm{Ir}$ & $\mathrm{Pt}^{*}$ & $\mathrm{Au}^{*}$ & $\mathrm{Hg}$ \\
\hline & & 4.23 & 4.10 & 3.98 & 3.87 & 3.76 & 3.65 & 3.54 & & & 3.24 \\
\hline & & & & & & & & & 3.77 & 3.66 & \\
\hline & (HF) & 4.26 & 4.07 & 3.92 & 3.80 & 3.69 & 3.60 & 3.52 & 3.76 & 3.70 & 3.33 \\
\hline
\end{tabular}

No attempt was made to calculate HF wave functions from our results, but the correspondence between $n s$ alkali expectation values [16], $P(r) / \bar{r}$, with $\sqrt{\rho}$, measured at the wave crests, demonstrates the feasibility of such a simulation, in Table 6.

\section{Ionization Radii}

The effect of applied pressure on the electronic structure of the hydrogen atom has been studied many times $[20,21]$ by changing the boundary condition in wavemechanical simulation of the energy-level structure. The general effect is an increase 
Table 5 Radial distances of highest inner-transition element maxima

\begin{tabular}{|c|c|c|c|c|c|c|c|}
\hline $\mathrm{Cs}$ & $\sigma$ & $\mathrm{La}^{*}$ & $\mathrm{Ce}$ & $\mathrm{Pr}$ & $\mathrm{Nd}$ & $\mathrm{Pm}$ & $\mathrm{Sm}$ \\
$r_{n}^{\prime \prime}$ & 0.825 & & 5.10 & 5.05 & 5.00 & 4.95 & 4.90 \\
6.31 & 0.775 & 4.89 & & & & & \\
& $(\mathrm{HF})$ & 4.93 & 5.11 & 5.05 & 4.99 & 4.93 & 4.88 \\
\hline $\mathrm{Eu}$ & $\mathrm{Gd}^{*}$ & $\mathrm{~Tb}$ & $\mathrm{Dy}$ & $\mathrm{Ho}$ & $\mathrm{Er}$ & $\mathrm{Tm}$ & $\mathrm{Yb}$ \\
4.86 & & 4.76 & 4.71 & 4.67 & 4.62 & 4.57 & 4.53 \\
& 4.56 & & & & & & \\
4.83 & 4.55 & 4.74 & 4.70 & 4.66 & 4.62 & 4.58 & 4.54 \\
\hline \hline $\mathrm{Fr}$ & & $\mathrm{Ac}^{*}$ & $\mathrm{Th}^{* *}$ & $\mathrm{~Pa}^{*}$ & $\mathrm{U}^{*}$ & $\mathrm{~Np}^{*}$ & $\mathrm{Pu}$ \\
$r_{n}^{\prime}$ & 0.75 & & & 5.11 & 5.06 & 5.01 & \\
6.95 & 0.795 & & & & & & 5.21 \\
& $(\mathrm{HF})$ & 5.23 & 4.98 & 5.11 & 5.05 & 5.00 & 5.18 \\
\hline $\mathrm{Am}$ & $\mathrm{Cm} *$ & $\mathrm{Bk}$ & $\mathrm{Cf}$ & $\mathrm{Es}$ & $\mathrm{Fm}$ & $\mathrm{Md}$ & $\mathrm{No}$ \\
& 4.86 & & & & & & \\
5.15 & & 5.05 & 5.00 & 4.95 & 4.91 & 4.86 & 4.81 \\
5.13 & 4.86 & 5.05 & 5.01 & 4.97 & 4.93 & 4.90 & 4.86 \\
\hline \hline
\end{tabular}

Table 6 Simulated wave functions

$$
\begin{array}{cccccc} 
& \mathrm{Li} & \mathrm{Na} & \mathrm{K} & \mathrm{Rb} & \mathrm{Cs} \\
|\psi(\mathrm{HF})| & 0.1352 & 0.1140 & 0.0840 & 0.0782 & 0.0666 \\
\sqrt{\rho} & 0.1844 & 0.1122 & 0.0917 & 0.0656 & 0.0566
\end{array}
$$

of all energy levels with pressure, until the point is reached where the ground-state level reaches the ionization limit on compression to a radius of $r_{0}=1.835 a_{0}$.

Such a calculation for non-hydrogen atoms was carried out numerically by a modified Hartree-Fock-Slater procedure [22]. The boundary condition for each wave function was introduced on defining a cut-off radius by the step function:

$$
S=e^{-\left(r / r_{0}\right)^{p}} \quad, \quad p>>1 .
$$

The value of $p$ determines the sharpness of the cut-off and it may vary from atom to atom.

A set of ionization radii with $p=20$ was found to correspond fairly well with the characteristic atomic radii [7] that generate chemical bond dissociation energies in either point-charge or Heitler-London simulation. The value of these characteristic radii are plagued by uncertainties in thermochemical quantities and in their relationship with spectroscopic measurements.

The rationale behind this identification lies therein that the energy simulations assume uniform one-electron density within the characteristic volume, whereas an electron, decoupled from the nucleus by hydrostatic compression, is likewise confined to a sphere of radius $r_{0}$ at constant density. By exploiting this property, ionization radii were also calculated from the maxima of HFS wave functions normalized over spheres of constant density [23]. The same procedure now suggests itself for 
the calculation of such radii, directly from the calculated charge densities $(\rho)$ and radial expectation values $\bar{r}$, in Fig. 7.

Normalization of the radial wave function in the ionization sphere requires

$$
\frac{4 \pi}{3} r_{0}^{3}\left(\frac{u^{2}(r)}{4 \pi}\right)=1
$$

The density at the radial maximum is given by: $\rho=\left(\bar{r} / r^{\prime \prime}\right)\left[1 / u^{2}(\bar{r})\right]$, and hence, in $\AA$ units,

$$
r_{0}=S\left(\frac{\bar{r}}{r_{n}^{\prime \prime}} \cdot \frac{3}{\rho}\right)^{\frac{1}{3}} a_{0} .
$$

The scale factor increases with the size of the excluded core region. In general

$$
S=\left(1-\frac{r_{c}}{r^{\prime \prime}}\right)
$$

for a core of radius $r_{c}$. For group $2, r_{c}=0$ and $S=1$. For higher groups $r_{c}=2 \bar{r}_{n-2}$. For the Na group only the $1 s$ level is inaccessible, hence $S=1-0.272 / 4.5=0.94$. In period 4 the appearance of a $d$-level between $\mathrm{Ca}$ and $\mathrm{Ga}$ results in contraction of the core, compensated for by setting $r_{c}=\bar{r}_{n-2}$, i.e. $S=0.93$ for the $4 p$ level. The results for representative elements of the $s$ and $p$ blocks are shown in Table 7 .

Table 7 Ionization radii of representative elements

\begin{tabular}{|c|c|c|c|c|c|c|c|c|c|c|}
\hline$n$ & \multirow{2}{*}{$r^{\prime \prime}$} & $\rho_{n}$ & \multicolumn{8}{|c|}{ Atom } \\
& & & \multicolumn{10}{|c|}{} \\
\hline 2 & 4.05 & 0.034 & $\mathrm{Li}$ & $\mathrm{Be}$ & $\mathrm{B}$ & $\mathrm{C}$ & $\mathrm{N}$ & $\mathrm{O}$ & $\mathrm{F}$ & $\mathrm{Ne}$ \\
& & $r_{0} / \AA$ & 2.36 & 2.20 & 1.88 & 1.78 & 1.69 & 1.60 & 1.52 & 1.44 \\
& & $r_{\text {emp }}$ & 2.70 & 1.70 & 1.85 & 1.85 & 1.62 & 1.51 & 1.37 & \\
& & $r_{0}(\mathrm{HF})$ & 1.25 & 1.09 & 1.62 & 1.60 & 1.56 & 1.45 & 1.36 & 1.20 \\
\hline 3 & 4.50 & 0.0126 & $\mathrm{Na}$ & $\mathrm{Mg}$ & $\mathrm{Al}$ & $\mathrm{Si}$ & $\mathrm{P}$ & $\mathrm{S}$ & $\mathrm{Cl}$ & $\mathrm{Ar}$ \\
& & $r_{0} / \AA$ & 3.09 & 2.87 & 2.74 & 2.62 & 2.51 & 2.47 & 2.30 & 2.21 \\
& & $r_{\text {emp }}$ & 3.00 & 2.10 & 2.60 & 2.90 & 2.81 & 2.66 & 2.30 & \\
& & $r_{0}(\mathrm{HF})$ & 2.73 & 2.35 & 2.61 & 2.40 & 2.20 & 2.05 & 1.89 & 1.81 \\
\hline 4 & 5.07 & 0.0084 & $\mathrm{~K}$ & $\mathrm{Ca}$ & $\mathrm{Ga}$ & $\mathrm{Ge}$ & $\mathrm{As}$ & $\mathrm{Se}$ & $\mathrm{Br}$ & $\mathrm{Kr}$ \\
& & $r_{0} / \AA$ & 3.50 & 3.08 & 3.00 & 2.89 & 2.80 & 2.71 & 2.61 & 2.52 \\
& & $r_{\text {emp }}$ & 3.74 & 2.90 & 2.10 & 3.00 & 2.92 & 2.90 & 2.59 & \\
& & $r_{0}(\mathrm{HF})$ & 3.74 & 3.26 & 3.29 & 2.94 & 2.62 & 2.40 & 2.28 & 2.12 \\
\hline 5 & 5.67 & 0.0043 & $\mathrm{Rb}$ & $\mathrm{Sr}$ & $\mathrm{In}$ & $\mathrm{Sn}$ & $\mathrm{Sb}$ & $\mathrm{Te}$ & $\mathrm{I}$ & $\mathrm{Xe}$ \\
& & $r_{0} / \AA$ & 3.81 & 3.54 & 3.31 & 3.20 & 3.09 & 2.98 & 2.88 & 2.75 \\
& & $r_{\text {emp }}$ & 4.10 & 3.43 & 2.90 & 2.80 & 3.40 & 3.30 & 2.92 & \\
& & $r_{0}(\mathrm{HF})$ & 4.31 & 3.83 & 3.55 & 3.26 & 3.01 & 2.81 & 2.60 & 2.49 \\
\hline 6 & 6.31 & 0.00323 & $\mathrm{Cs}$ & $\mathrm{Ba}$ & $\mathrm{Tl}$ & $\mathrm{Pb}$ & $\mathrm{Bi}$ & $\mathrm{Po}$ & $\mathrm{At}$ & $\mathrm{Rn}$ \\
& & $r_{0} / \AA$ & 4.03 & 3.75 & 3.43 & 3.32 & 3.22 & 3.12 & 3.03 & 2.93 \\
& & $r_{\text {emp }}$ & 4.30 & 3.74 & 2.80 & 3.10 & 3.19 & 3.50 & & \\
& & $r_{0}(\mathrm{HF})$ & 4.96 & 4.48 & 3.82 & 3.47 & 3.19 & 3.14 & 3.12 & 3.82 \\
\hline
\end{tabular}


Ionization radii calculated with the same formula and scale factors $S(4)=0.88$, $S(5)=0.81$ and $S(6)=0.78$ correspond well with the values calculated by atomic compression, $r_{0}$ (HFS), for the three $d$-series, as tabulated in Table 8 .

Table 8 Ionization radii of $d$-block elements

\begin{tabular}{|c|c|c|c|c|c|c|c|c|c|c|}
\hline$r_{0}$ & $\mathrm{Sc}$ & $\mathrm{Ti}$ & $\mathrm{V}$ & $\mathrm{Cr}$ & $\mathrm{Mn}$ & $\mathrm{Fe}$ & $\mathrm{Co}$ & $\mathrm{Ni}$ & $\mathrm{Cu}$ & $\mathrm{Zn}$ \\
$r_{0} / \AA$ & 3.02 & 2.99 & 2.96 & 3.02 & 2.90 & 2.87 & 2.85 & 2.81 & 2.88 & 2.76 \\
$r_{0}(\mathrm{HF})$ & 3.13 & 3.01 & 2.95 & 2.98 & 2.94 & 2.87 & 2.85 & 2.86 & 2.85 & 2.78 \\
\hline & $\mathrm{Y}$ & $\mathrm{Zr}$ & $\mathrm{Nb}$ & $\mathrm{Mo}$ & $\mathrm{Tc}$ & $\mathrm{Ru}$ & $\mathrm{Rh}$ & $\mathrm{Pd}$ & $\mathrm{Ag}$ & $\mathrm{Cd}$ \\
$r_{0} / \AA$ & 3.27 & 3.24 & 3.30 & 3.26 & 3.14 & 3.20 & 3.16 & 2.29 & 3.11 & 3.00 \\
$r_{0}(\mathrm{HF})$ & 3.55 & 3.32 & 3.30 & 3.21 & 3.16 & 3.13 & 3.08 & 2.49 & 3.04 & 3.02 \\
\hline & $\mathrm{Lu}$ & $\mathrm{Hf}$ & $\mathrm{Ta}$ & $\mathrm{W}$ & $\mathrm{Re}$ & $\mathrm{Os}$ & $\mathrm{Ir}$ & $\mathrm{Pt}$ & $\mathrm{Au}$ & $\mathrm{Hg}$ \\
$r_{0} / \AA$ & 3.54 & 3.50 & 3.47 & 3.44 & 3.40 & 3.37 & 3.34 & 3.41 & 3.38 & 3.24 \\
$r_{0}(\mathrm{HF})$ & 4.24 & 3.83 & 3.57 & 3.42 & 3.38 & 3.37 & 3.23 & 3.16 & 3.14 & 3.12 \\
\hline
\end{tabular}

Ionization radii for the lanthanides are in Table $9,(S=0.97)$.

Table 9 Ionization radii of the lanthanides

\begin{tabular}{|c|c|c|c|c|c|c|c|c|}
\hline Atom & \multicolumn{2}{|l|}{} & $\mathrm{La}$ & $\mathrm{Ce}$ & $\mathrm{Pr}$ & $\mathrm{Nd}$ & $\mathrm{Pm}$ & $\mathrm{Sm}$ \\
$r_{0} / \AA$ & \multicolumn{2}{|c}{} & 4.62 & 4.69 & 4.68 & 4.66 & 4.64 & 4.63 \\
$r_{0}(\mathrm{HF})$ & & 4.13 & 4.48 & 4.53 & 4.60 & 4.56 & 4.56 \\
\hline Atom & $\mathrm{Eu}$ & $\mathrm{Gd}$ & $\mathrm{Tb}$ & $\mathrm{Dy}$ & $\mathrm{Ho}$ & $\mathrm{Er}$ & $\mathrm{Tm}$ & $\mathrm{Yb}$ \\
$r_{0} / \AA$ & 4.61 & 4.51 & 4.58 & 4.57 & 4.55 & 4.54 & 4.52 & 4.50 \\
$r_{0}(\mathrm{HF})$ & 4.60 & 4.22 & 4.59 & 4.56 & 4.63 & 4.63 & 4.62 & 4.66 \\
\hline
\end{tabular}

Ionization radii are of fundamental importance in chemistry. By definition they represent the volume to which activated valence electrons are confined, and hence the quantum-potential energy of the valence state. This quantity is the same as the classical concept of electronegativity [24]. Not only is the entire theory of chemical reactivity entangled with electronegativity, but the ionization sphere also features directly in the simulation of interatomic interactions. Previous efforts to model ionization radii theoretically invariably involved some unsubstantiated assumptions. The present calculation proceeds without such assumptions, from derived extranuclear electronic arrangements.

\section{Discussion}

The calculation of atomic structure presented here assumes a wave structure of extranuclear electronic charge,distributed in a way self-similar to prominent objects such as planetary, solar and galactic systems, as observed in local three-dimensional space. Experience shows that all structures of this type are correctly simulated as 
a process of optimization based on golden spirals. For atoms a divergence angle of $\phi=4 \pi /(2 n-1)$ predicts a spherical wave structure of period $n^{2} a$, as derived before with the Bohr model of the hydrogen atom. To be consistent with the observed symmetry of the periodic table a well-defined shell structure that allows direct calculation of charge density is inferred. The remarkable result, for a unitary atom, is a charge distribution that scales linearly to the famous Thomas-Fermi statistical distribution, considered valid for all atoms. Whereas a TF calculation involves a sophisticated numerical procedure that depends on a critical selection of initial slope for the density curve [14], the new calculation amounts to a simple computation performed directly with a pocket calculator.

On superposition of the implied wave structure the TF-like arrangement is transformed into a periodic curve that now resembles a distribution with the same periodic structure as a typical HF simulation of a many-electron unitary atom. To bring this result into register with actual HF models only needs a set of screening constants that regulates contraction of the density function in the field of a nuclear charge of $+Z$ e. Rather than random variables, these screening constants are small numbers that reflect a variability commensurate with the periodic table.

From a chemical point of view the outer maximum in charge density, which represents the valence density, is the most important aspect of the entire charge distribution. Having simulated the effect of compression on the valence density by HFS SCF methods myself, I am aware of the effort involved. Being able to perform, what is clearly a superior simulation, with only a pocket calculator and no further assumptions, convinced me that the heavy computations of modern quantum chemistry are not needed.

The simple reason for this is now well established: Quantum mechanics, like relativity, is the non-classical theory of motion in four-dimensional space-time. All theories, formulated in three-dimensional space, which include Newtonian and wave mechanics, are to be considered classical by this criterion. Wave mechanics largely because it interprets elementary matter, such as electrons, as point particles, forgetting that the motion of particulate matter needs to be described by particle (Newtonian) dynamics. TF and HF simulations attempt to perform a wave-like analysis and end up with an intractable probability function.

On assuming an electronic wave structure the problem is simplified by orders of magnitude, using elementary wave mechanics. Calculations of this type are well within the ability of any chemist without expertise in higher mathematics. It has already been shown [25] that the results reported here define a covalence function that predicts, without further assumption, interatomic distances, bond dissociation energies, and harmonic force constants of all purely covalent interactions, irrespective of bond order. In line with the philosophy that molecular shape, as a classical concept, should be modelled classically, the ultimate aim is to derive the principles that underlie molecular structure and to outline a fundamental system of molecular mechanics.

Perhaps the most important result of the calculation is the way in which it confirms the number-theoretic structure of the periodic table, shown in Fig. 3, and the wave structure of the electron. From the atomic model shown in Fig. 7, the volume 
of each segment of Fig. 3 is calculated directly in units of $a^{3}$. The results are shown in Table 10.

Table 10 Relative volumes, $V_{e}$ (in units of $a^{3} \equiv a_{0}^{3}$ on the $H$-scale) of unit-electron wave packets at various atomic sub-levels. $V_{T}$ is the calculated volume of periodic standing waves confined to spherical annuli. All $\left(V_{e}\right)$ are conveniently expressed as $100 \tau^{m}$, within rounding-off errors

\begin{tabular}{|c|c|c|c|c|c|c|c|c|c|}
\hline$P$ & $s^{2}$ & $r_{s}$ & $f^{14}$ & $d^{10}$ & $p^{6}$ & $r_{p}$ & $V_{T}$ & $\sum e$ & Legend \\
\hline 1 & $\begin{array}{c}29.3 \\
(15) \\
\tau^{4}\end{array}$ & 1.52 & & & & & $\begin{array}{c}29.3 \\
(15) \\
\tau^{4}\end{array}$ & 2 & $\begin{array}{c}V_{\text {sub }} \\
\left(V_{e}\right) \\
V_{e} / 100\end{array}$ \\
\hline$x$ & $\Phi^{2}$ & & & & & & $\Phi^{13 / 3}$ & & \\
\hline 2 & $\begin{array}{c}77 \\
(38) \\
\tau^{2}\end{array}$ & 2.09 & & & $\begin{array}{c}166 \\
(28) \\
\tau^{\frac{8}{3}}\end{array}$ & 1.88 & $\begin{array}{c}235 \\
(30) \\
\tau^{\frac{8}{3}}\end{array}$ & 8 & \\
\hline$x$ & $\Phi^{2}$ & & & & $\Phi^{2}$ & & $\Phi^{2}$ & & \\
\hline 3 & $\begin{array}{c}201 \\
(100) \\
\tau^{0}\end{array}$ & 2.88 & & & $\begin{array}{c}436 \\
(73) \\
\tau^{\frac{2}{3}}\end{array}$ & 2.59 & $\begin{array}{c}637 \\
(80) \\
\tau^{\frac{1}{2}}\end{array}$ & 8 & \\
\hline$\times$ & $\Phi$ & & & & $\Phi$ & & $\Phi^{5 / 2}$ & & \\
\hline 4 & $\begin{array}{c}326 \\
(162) \\
\Phi\end{array}$ & 3.38 & & $\begin{array}{l}1120 \\
(112) \\
<\Phi^{\frac{1}{3}}\end{array}$ & $\begin{array}{c}705 \\
(118) \\
\Phi^{\frac{1}{3}}\end{array}$ & 3.04 & $\begin{array}{c}2151 \\
(120) \\
\Phi^{\frac{1}{3}}\end{array}$ & 18 & \\
\hline$x$ & $\Phi$ & & & $\Phi^{5 / 3}$ & $\Phi$ & & $\Phi^{3 / 2}$ & & \\
\hline 5 & $\begin{array}{c}527 \\
(262) \\
\Phi^{2}\end{array}$ & 3.97 & & $\begin{array}{l}2517 \\
(252) \\
<\Phi^{2}\end{array}$ & $\begin{array}{c}1141 \\
(190) \\
\Phi^{\frac{4}{3}}\end{array}$ & 3.56 & $\begin{array}{c}4185 \\
(233) \\
\Phi^{\frac{7}{4}}\end{array}$ & 18 & \\
\hline$x$ & $\Phi$ & & & $\Phi^{2 / 3}$ & $\Phi$ & & $\Phi^{7 / 4}$ & & \\
\hline 6 & $\begin{array}{c}853 \\
(424) \\
\Phi^{3}\end{array}$ & 4.66 & $\begin{array}{c}3668 \\
(252) \\
\Phi^{2}\end{array}$ & $\begin{array}{c}3552 \\
(355) \\
\Phi^{\frac{8}{3}}\end{array}$ & $\begin{array}{c}1846 \\
(308) \\
\Phi^{\frac{7}{3}}\end{array}$ & 4.19 & $\begin{array}{c}9919 \\
(310) \\
\Phi^{\frac{7}{3}}\end{array}$ & 32 & \\
\hline
\end{tabular}

All results derive from the volume of the inner shell of two electrons, $V_{1}=$ $29.375 a^{3}$. Higher 2-electron sub-levels (called $s$ ) and $p$-levels increase in size by factors of $\Phi^{x}$ with $x=1$ or 2 , as shown in the multiplication rows. The factors that relate the $d$-level volumes are fractional powers of $\Phi$, due to the irregular occupation numbers at these levels. The volume of a single $s$ electron at the second level follows as $38=100 \tau^{2} / a^{3}$. To generate the complete table from this value we only have to assume a volume of $100 \tau^{\frac{8}{3}} / a^{3}$ for a second period $p$-electron. The average one-electron volume increases steadily from $100 \tau^{4}$ for period 1 to $100 \Phi^{3}$ for period 6. The factor 100 is consistent with the identification of 100 natural elements [6].

We notice two further potentially meaningful trends. To good approximation the average volume per electron $\left(V_{e}\right)$ increases in even multiples of $V_{1} / 6$, with increas- 
ing periodic level $P$, from $3 V_{1} / 6$ to $32 V_{1} / 3$ at $V_{6} \simeq V_{1} \times \Phi^{12} \simeq 100^{2}$. Also, a regular decrease at $P=6$ of $V_{e} / 100$ from $\Phi^{3}\left(\equiv \Phi^{\frac{9}{3}}\right)$ to $\Phi^{2}$ in the sequence $s>d>p>f$.

These results can only be rationalized by considering an electron as a flexible wave packet, rather than a point particle. In particular, as shown in Table 2, the volume of a given annular shell is reduced as the nuclear charge increases, which means that an electronic unit is compressed into less space. However, the effective dimensionless electronic radius of $r / a$ remains constant as $a$ decreases and defines the fine-structure constant as $\alpha=\sqrt{r / a}$. The dimensionless volume of the twoelectron $(1 s)$ annulus therefore remains constant and so does the effective separation of electrons in units of $a$.

Table 11 Mean empirical radii (from Table 3) of p-electrons at different levels, compared to calculated electronic radii $\left(r_{e}\right)$ of the electrons, considered to be spherical waves(Table 10). All radii in units of $a$.

\begin{tabular}{|c|c|c|c|c|c|}
\hline $\mathrm{P}$ & 2 & 3 & 4 & 5 & 6 \\
\hline $\bar{r}_{e m p}$ & 1.43 & 2.33 & 2.50 & 2.92 & 3.11 \\
\hline$r_{e}=\left(\frac{3 V_{e}}{4 \pi}\right)^{\frac{1}{3}}$ & 1.88 & 2.59 & 3.04 & 3.56 & 4.19 \\
\hline$\frac{3}{4}\left(\bar{r} / r_{e}\right)$ & 0.58 & 0.67 & 0.64 & 0.67 & 0.68 \\
\hline$\sigma$ & 0.59 & 0.8 & 0.7 & 0.73 & 0.68 \\
\hline$\mu$ & 1.17 & 1.14 & 1.11 & 1.11 & 1.10 \\
\hline$\left(\frac{4 \pi}{r_{e}}\right)^{\frac{1}{12}}$ & 1.17 & 1.14 & 1.13 & 1.11 & 1.10 \\
\hline
\end{tabular}

The effect of atomic compression is now also better understood in view of the different unit volumes at different sub-levels, which correlate with orbital angular momentum. To resist compression the large $s$ electrons are forced into higher levels relative to $p, d$ and $f$ electrons. By comparison, an $f$ electron absorbs compressive energy by spinning more rapidly in confined space. Total inversion of relative energy towards $f<d<p<s$ must result at high pressure.

As shown in Table 11, mean electronic radii at various $p$-levels correlate reasonably well with the mean empirically estimated radii and the parameters $\sigma$ and $\mu$ of Table 3. The estimate of $\mu=\left(4 \pi / r_{e}\right)^{\frac{1}{12}}$ derives from spherical contraction due to increased nuclear charge, proportional to $1 / r^{2}$, over 6 steps. These estimates only serve to show that the empirical parameters do not vary randomly.

\section{Concluding Summary}

Nucleogenesis in the interior of massive stellar objects yields 100 natural elements of composition $Z /(A-Z)=1$. Because of radioactive decay at reduced pressure in 
intergalactic space the stability ratio converges as a function of mass number to a value of $\tau$ at $A=267=(A-Z) \Phi=Z \Phi^{2}$. As a result only 81 stable elements survive in the solar system as a periodic array conditioned by $\tau$. The observed periodicity corresponds to a Ford-circle mapping of the fourth order unimodular Farey sequence of rational fractions.

The same periodic function results from optimization on a golden spiral with a variable convergence angle of $4 \pi /(2 n-1)$, which describes a spherical standing wave with nodes at $n^{2}$. Analysis of the wave structure shows that it correctly models the atomic electron distribution for all elements as a function of the golden ratio and the Bohr radius, $a_{0}$. Normalization of the wave structure into uniform spherical units simulates atomic activation, readily interpreted as the basis of electronegativity and chemical affinity.

The same model is shown to fit the electronic structure of all atoms when described in dimensionless units. The scaling symmetry observed here obeys the symmetry law of Haüy (1815), quoted by Janner [26]:

Symmetry consists in a repeated decreasing of an object in such a way that by changing the visual distance it looks the same.

It is also known as self-similarity, a concept which is intimately related to the golden ratio, and known to operate on a cosmic scale. Our observations may therefore be rationalized by considering elementary matter as the product of large-scale space-time curvature, as described by the golden ratio. We reach the provocative conclusion that a construct, which is entirely governed by the properties of the golden ratio and number theory, predicts the electronic configuration of all atoms, without reference to any chemical know-how, as a basis of a chemical theory. 


\section{References}

1. Boeyens (2012) Chemistry in 4 Dimensions. This volume.

2. Boeyens JCA (2010) Int J Mol Sci 11:4267.

3. Boeyens JCA (2009), Physics Essays 22:493.

4. Harkins WD (1931), Phys Rev 38:1270.

5. Boeyens JCA (2003) J Radioanal Nucl Chem 259:33.

6. Boeyens JCA, Levendis DC (2008), Number Theory and the Periodicity of Matter. Springer.com.

7. Boeyens JCA (2008) Chemistry from First Principles. Springer.com.

8. Boeyens JCA, Comba P (2012). This volume.

9. Coxeter HSM (1989) Introduction to Geometry. 2nd ed. Wiley, NY.

10. Dunlap RA (1997) The Golden Ratio and Fibonacci Numbers. World Scientific, Singapore, p. 20 .

11. Veblen O (1933) Projektive Relativitätstheorie. Springer-Verlag, Berlin. English translation in [12].

- Hoffman B (1930) Phys Rev 36:810.

12. Boeyens JCA (2010) Chemical Cosmology. Springer.com.

13. Boeyens (2003) The Theories of Chemistry. Elsevier, Amsterdam.

14. Condon EU, Odabaşi H (1980) Atomic Structure. University Press, Cambridge.

15. Hinchliffe A (1999) Chemical Modeling from Atoms to Liquids. Wiley, N.Y. p. 200.

16. Mann JB (1968) Atomic Structure Calculations II. Los Alamos Scientific Report, LA-3691.

17. Schrödinger E (1937) Nature, 140:742.

18. Madelung E (1926) Z für Physik 40:322.

19. Pauli W, Weisskopf V (1934)Helv phys acta 7:709.

20. Sommerfeld A, Welker H (1938) Ann Phys 424:56.

21. Goldman S, Joslin C (1992) J Phys Chem 96:6021.

22. Boeyens JCA (1994) J Chem Soc Faraday Trans 90:3377.

23. Boeyens JCA (1978) S Afr J Chem 31:121.

24. Boeyens JCA (2008) Z Naturforsch 63b:199.

25. Papers in this volume.

26. Janner A (2001) Crystal Engin 4:119. 